\title{
THE FUTURE OF SPACECRAFT NUCLEAR PROPULSION
}

\author{
F. JANSEN*, ON BEHALF OF THE MEGAHIT AND DIPOP CONSORTIA ${ }^{\dagger}$ \\ *DLR Institute of Space Systems, 28359 Bremen, Robert-Hooke-Str.7, Germany, \\ E-mail: frank.jansen@dlr.de
}

\begin{abstract}
This paper summarizes the advantages of space nuclear power and propulsion systems. It describes the actual status of international power level dependent spacecraft nuclear propulsion missions, especially the high power EU-Russian MEGAHIT study including the Russian Megawatt-Class Nuclear Power Propulsion System, the NASA GRC project and the low and medium power EU DiPoP study. Space nuclear propulsion based mission scenarios of these studies are sketched as well.
\end{abstract}

\section{Introduction}

The space nuclear power respectively propulsion systems have the following three main advantages:

1) the available power / propulsion energy never depends on spacecraft orientation, on Sun lighting in Earth orbit or in the heliosphere,

2) the nuclear power systems in space are feasible and

3 ) in comparison with solar energy high-power systems, there are essential advantages: a) smaller mass, dimensions and dynamic characteristics, b) radiation resistance in exploitation and c) the feasibility to use for extended time periods, high-efficient plasma thrusters.

The result of the advantages for manned and unmanned space flights - the high mass, the fast and independent speed, allows one to realize nearby Earth-Moon, asteroid, inner and outer planet as well as deep space interstellar missions and habitat projects.

\section{Power Level Dependent Spacecraft Nuclear Propulsion Missions}

Three classes of nuclear electrical propulsion are studied internationally - the 30kWe, 200 - 300kWe and the MWe power range. The highest range is covered by EU-Russian MEGAHIT (Megawatt Highly Efficient Technologies for Space Power and Propulsion Systems for Long-duration Exploration Missions) and NASA GRC teams and the medium respectively low power one was in priority

\footnotetext{
${ }^{\dagger}$ Work supported by MEGAHIT and DIPOP study teams. See also under acknowledgement below.
} 
of the EU DiPoP (Disruptive technologies for space Power and Propulsion) and NASA GRC feasibility studies.

MEGAHIT is a supporting action, in preparation of the European Union Horizon 2020 programme. Its objective is to propose specific actions and a roadmap in the field of MWe nuclear electric propulsion (www.megahit-eu.org). A European community including Russian partners around nuclear space power systems and an analysis of the potential international collaboration opportunities is carried out (J.-C. Worms et al. [1]). Especially from the following seven topics, the technological priorities for the realization of an international nuclear space mission are identified: 1 . fuel and core choice, related to practicability of space nuclear technologies and proper shielding, 2. thermal control including addressing heat transportation and radiating devices layout, 3. energy conversion from thermal energy into electricity at high power level, 4. propulsion with respect to applicable electric thrusters technologies, 5. electric power management and distribution related to the high power converters and distribution cables between the generator and consumers of the spacecraft, 6 . structure and spacecraft arrangement, which includes the space system architecture and usage of lightweight structures material and 7. safety regulations, especially addressing the nuclear safety, public acceptance and other regulations.

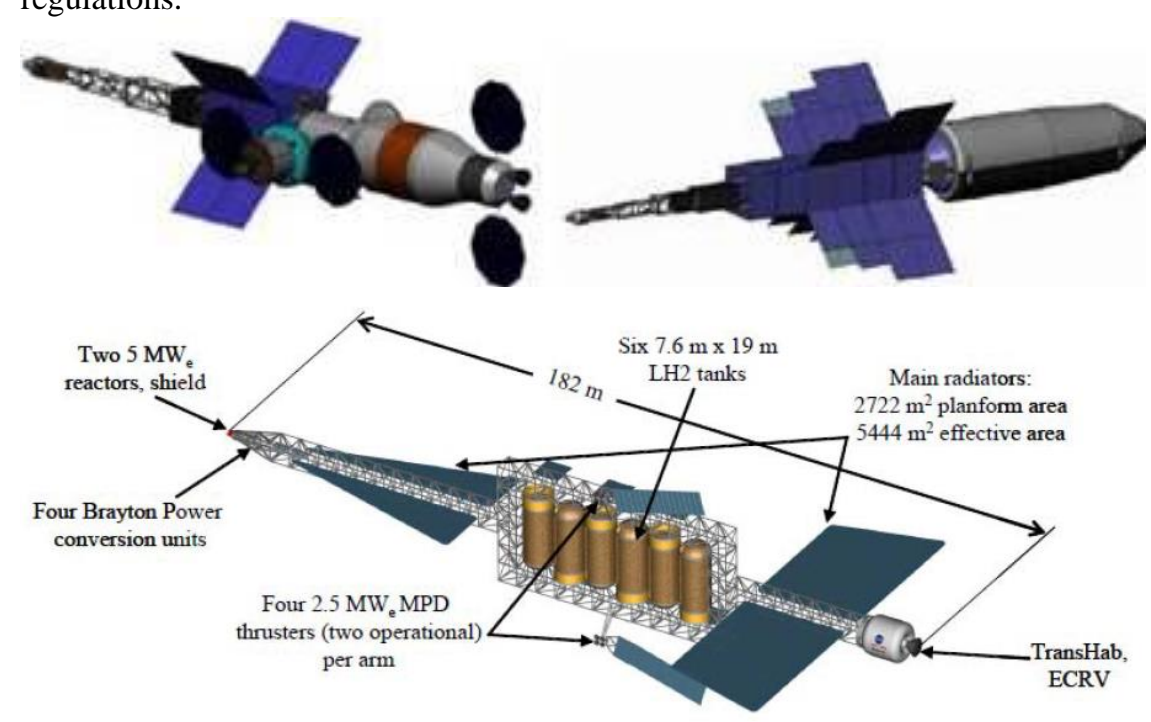

Figure 1 Current NASA nuclear electric propulsion vehicles: human NEO mission (upper left). M.L. McGuire et al [2] published the NASA GRC Mars-2033 MW class nuclear electric propulsion systems. The upper line right displayed the Mars cago transfer vehicle (CTV) and the lower line the Mars transfer vehicle (MTV). 
The CTV has an NEP system with 5 MWe electrical power and the crewed MTV has two reactors with total of $10 \mathrm{MW}$ electrical power. Both vehicles use a low-thrust, high-efficiency (5000 sec specific impulse) magnetoplasmadynamic (MPD) system with hydrogen as propellant to conduct a spiral-out of the Earth gravity well, a low-thrust heliocentric trajectory, and a spiral-in at Mars with arrival in 2033. The MTV is $182 \mathrm{~m}$ long and must be assembled in orbit.

An innovative project to create a Transport Power Module (TPM) based on a Megawatt-Class Nuclear Power Propulsion System (NPPS) is currently being developed in Russia (L.E. Zakharenkov et al. [3], see Figure 2). NPPS project has to be ready in 2018 with the ground testing, flight test preparation of TPM and a later robotic mounting of the entire system in a high Earth orbit for safety reasons (above $1200 \mathrm{~km}$ ). TPM will have one nuclear reactor of $3.5 \mathrm{MW}$ thermal power level, four closed gas-turbine Brayton cycle units for thermal power conversion and generating of $1 \mathrm{MWe}$. The power management and distribution system manage on-board power of about 1 MWe. NPPS will have high power ion thrusters of total power $\sim 1$ MWe with the Isp of about $7000 \mathrm{~s}$.

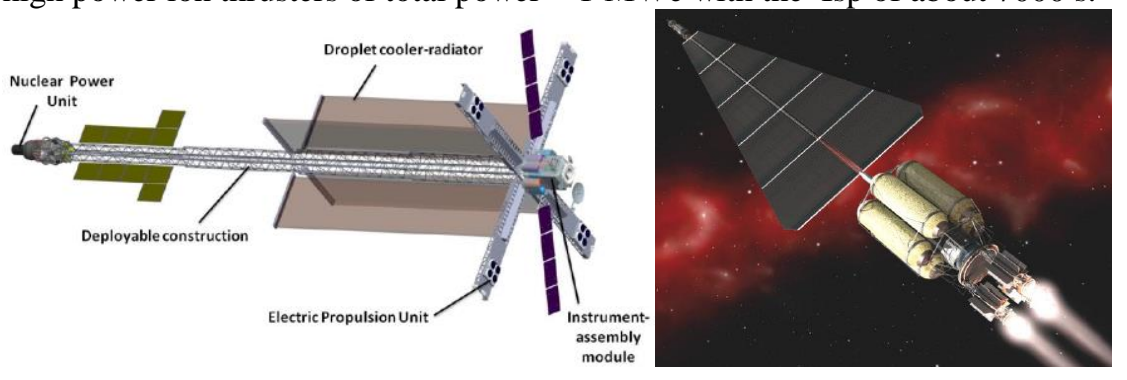

Figure 2 Left: main components of the Russian NPPS. Right: artist view of high power nuclear electric propulsion system (credits : CNES/Antigravite/A.Szames).

The EU DiPoP feasibility study (www.DiPoP.eu) from 2011-2012 selected the following range of potential space applications of NEP: a) removal of 'dead' spacecraft from Earth orbit to reduce space debris, b) high power ground penetrating radar, ice-melting laser, long distance high data rate communications, c) space port power generation on planetary surfaces, d) spacebased NEO tracking radar for trajectories determination obscured by the Sun, NEO deflection, survey and mining, e) a Mars human mission with chemical propulsion and nuclear powered habitat infrastructures on Mars surface, f) outer planet exploration like Jupiter sample return as well as Neptune orbital survey and lander and $\mathrm{g}$ ) heliosphere and beyond exploration.

The $30 \mathrm{kWe}$ prioritisations are planetary surface power generation and small robotic exploration. The $200 \mathrm{kWe}$ prioritisations are NEO deflection, survey, 
mining, outer planet robotic exploration and large infrastructure transportation. The DiPoP roadmap for low and medium nuclear power is presented in Figure 3.

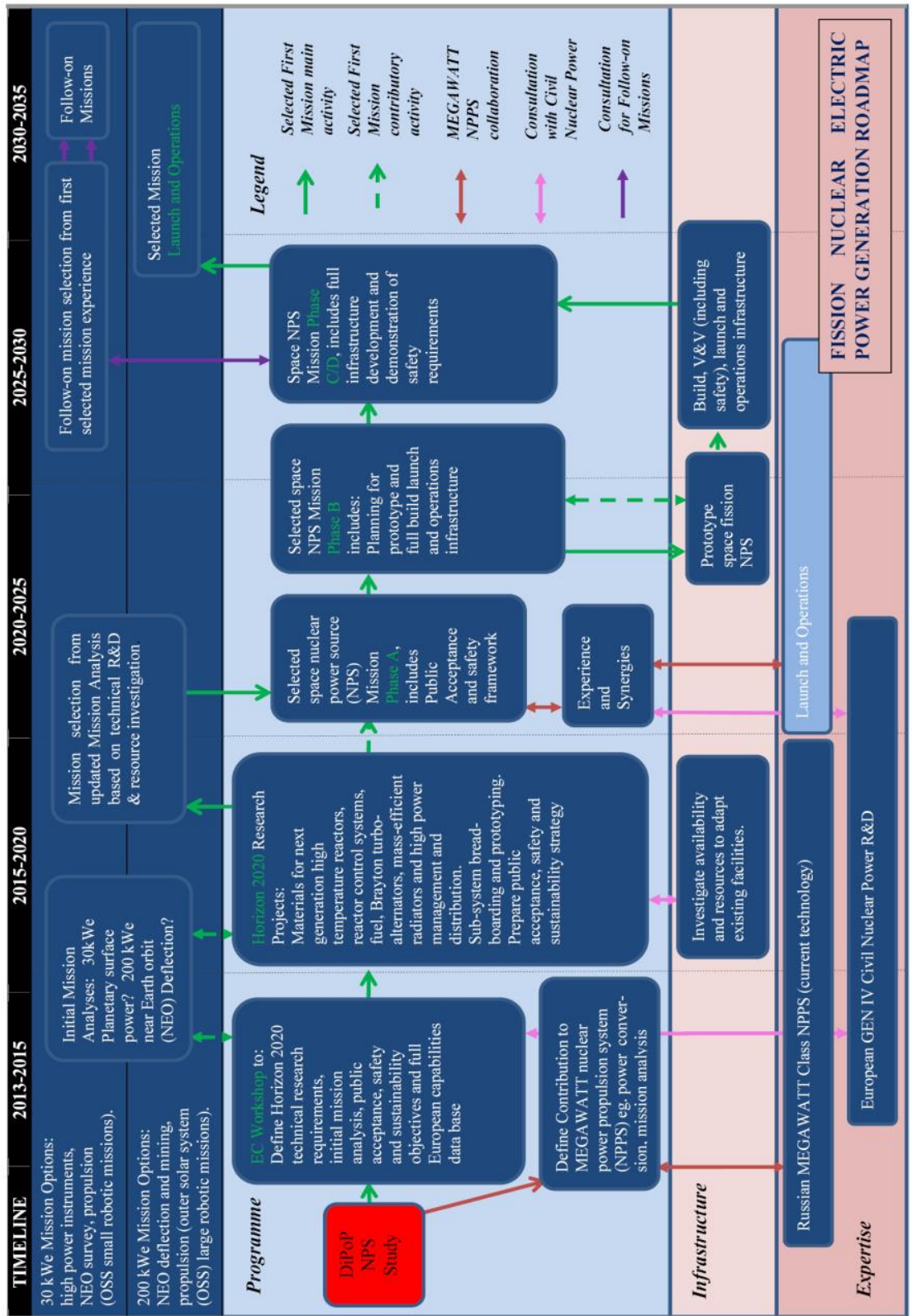

Figure 3 Explanations of the low and medium nuclear power European roadmap are published by DiPoP consortium under R. Blott et. al. [4] and [5]. 


\section{Summary}

The mentioned studies realize, that the future of spacecraft nuclear propulsion with applications to several space mission is under a very positive discussion of space organizations and space faring nations in Europe, Russia, the USA as well as for example in China, Brazil, South Africa and other countries. These countries are able and willing to contribute - under consideration of their economic, technological, scientific, public and political circumstances - to a third (after Apollo Moon landings and International Space Station), truly international and highly welcomed space mission project of mankind.

\section{Acknowledgments}

This paper is based on contributions from the EU funded projects MEGAHIT (GRANT agreement number 313096) with the team members J.-C. Worms, European Science Foundation, Strasbourg, France; Z. Hodgson National Nuclear Laboratory, Sellafield, UK; E. Cliquet, Centre National d'Etudes Spatiales, Paris, France; F. Jansen, The German Aerospace Center, Bremen, Germany; A. Semenkin, The State Scientific Centre "Keldysh Research Centre", Moscow, Russia; E. Gaia Thales Alenia Space, Torino, Italy) and DiPoP (GRANT agreement number 284081) with the team members Ch. Koppel, KopooS Consulting Ind., Paris, France; R. Blott, Space Enterprise Partnerships, Chichester, UK; F. Jansen, The German Aerospace Center, Bremen, Germany; G. Herdrich, University of Stuttgart, Germany; H. Kersten, University of Kiel, Germany and C. Ferrari, ISIS R\&D, Rome, Italy.

\section{References}

1. J.-C. Worms, E. Cliquet, E. Detsis, E. Gaia, F. Jansen, G. Poidomani, J.-M. Ruault, A. Semenkin, T. Tinsley, N. Walter, A. Koroteev, $64^{\text {th }}$ International Astronautical Congress, IAC-13-C4.7-C3.5, (2013).

2. M.L. McGuire et al, NASA/TM-2006-214106, (2006).

3. L.E. Zakharenkov, A.V. Semenkin and A.E. Solodukhin, $5^{\text {th }}$ European Conference for Aerospace Sciences (EUCASS), Munich, in press, (2013).

4. R. Blott, Ch. Koppel, D. Valentian, F. Jansen, C. Ferrari, C. Bruno, G. Herdrich, R. Gabrielli, , $64^{\text {th }}$ International Astronautical Congress, IAC13-C.4.7-C.3.5.4, (2013).

5. R. Blott, Ch. Koppel, D. Valentian, F. Jansen, C. Ferrari, C. Bruno, G. Herdrich, R. Gabrielli, "Is there a future for fission nuclear electric propulsion", presented at 33rd International Electric Propulsion Conference, Washington DC, IEPC-2013-325, (2013). 